\title{
Crítica y utopía en la filosofía de Franz Hinkelammert
}

\author{
Estela Fernández NadAl \\ CONICET / Universidad Nacional de Cuyo \\ Mendoza, Argentina
}

\section{La condición humana y la dialéctica de la emancipación}

Para Franz Hinkelammert, la historia de la humanidad es un largo proceso en el que, frente al poder instituido, producto de una determinada correlación de fuerzas sociales, emerge un sujeto que lo resiste, impulsando así la transformación histórica hacia una nueva configuración social, donde reaparecen formas de dominación e institucionalización nuevas.

Puede decirse que la condición humana es una paradójica e irresoluble tensión entre libertad y dominación. Por una parte, el ser humano sólo puede devenir tal a partir de su ingreso en un proceso de institucionalización. Las instituciones (Estado, mercado, matrimonio) son consecuencia del carácter fragmentario de las relaciones humanas y representan un límite de opacidad y finitud inherente a la propia condición humana. Sin ellas, el hombre quedaría limitado dentro de un espacio de experiencia reducida e inmediata. Por otra parte, por su propia lógica, las instituciones creadas para permitir el desarrollo humano, tienden a independizarse del hombre, a subordinarlo y a sacralizarse. A la larga, atentan contra la vida, la socavan. Las instituciones son, por tanto, necesarias y peligrosas. 
Esta concepción antropológica, implícita en la filosofía política de Hinkelammert, se articula con un enfoque histórico. Desde este punto de vista, en el desarrollo de la modernidad occidental, el proceso de emancipación humana, esto es, la lucha por la dignificación del hombre como sujeto de derechos, se plantea como el resultado del desenvolvimiento de dos impulsos emancipatorios sucesivos. La relación que guardan entre sí ambas emancipaciones no es de derivación continua, sino de negación dialéctica ${ }^{1}$.

Un primer momento en ese proceso corresponde a la emergencia y constitución de la legalidad capitalista, producto de las revoluciones burguesas de los siglos XVII y XVIII. Dentro de este horizonte, una primera determinación de la igualdad y la libertad es la que formula John Locke, representante intelectual de la gloriosa revolución inglesa, que legitimaría teóricamente el nuevo papel de Inglaterra como potencia imperial mundial. Locke concibe la libertad y la igualdad a partir del modelo del contrato de compra y venta, donde individuos particulares-abstractos intercambian bienes y servicios en el mercado. Es un concepto formal, abstracto, "contractual", de igualdad/libertad, pues el sujeto de derechos está despojado de su dimensión real, como sujeto corporal de necesidades, que deben ser satisfechas para que no perezca. Los derechos básicos a la integridad física, la dignidad personal, la propiedad como medio de subsistencia, son abolidos en función de un solo derecho: la propiedad acumulativa como sistema de eficiencia y competencia ${ }^{2}$.

Ahora bien, en este horizonte burgués de emancipación humana, despejado por la Revolución inglesa y teorizado por Locke, la Revolución Francesa viene a postular una categoría diferente de la igualdad/ libertad contractual. Es la categoría de "ciudadano", que procede de Rousseau y que, a diferencia de la de "individuo privado autónomo" de Locke, satisfecho en todas sus expectativas por el libre intercambio mercantil, porta una ambigüedad teórica profunda, capaz de reformular el sentido de la primera emancipación humana y de dinamizar un proceso libertario de otro signo.

Con la aparición del "ciudadano", como es sabido, la propiedad, lejos de ser suprimida, es legitimada, y la igualdad es establecida en el nivel impersonal de la ley, sin capacidad de injerencia en el espacio extrapolítico de la economía y la vida privada. Pero también es cierto que, en un cierto sentido, la supremacía del interés general, representado en el Estado, sugiere la atribución de una facultad de control 
público sobre las apetencias y pasiones privadas, de donde puede seguirse la regulación preventiva de la acumulación excesiva y de las relaciones mercantiles liberadas a su propia lógica.

De esta forma, la categoría de ciudadanía, al consagrar un espacio público al interior de la sociedad burguesa, desencadena una dinámica de la igualdad concreta, que da lugar a una segunda emancipación. El paradigma teórico de esta nueva exigencia de emancipación lo formula Marx, cuando devela la inversión que acontece en el seno de la igualdad contractual: la lógica de la compra y venta, que se muestra como realización de la igualdad en la esfera de la circulación, oculta la desigualdad real de los hombres en la órbita de la producción. La crítica marxista se realiza en nombre de la igualdad y dignidad humanas supuestamente requeridas y prometidas por la primera emancipación, para denunciar que no se han realizado en el marco de la sociedad burguesa. El descubrimiento de Marx de la reaparición de la dominación bajo un ropaje nuevo se extiende, durante los dos siglos posteriores, desde la lucha obrera por el socialismo hacia otros ámbitos de afirmación del sujeto humano concreto: por la emancipación de las mujeres, por la liberación de los esclavos, contra el racismo, por la independencia política de las colonias, por la preservación de la naturaleza, etc.

La nueva concepción del hombre que subyace a esta segunda emancipación produce un nuevo tipo de derechos humanos, que ahora se reclaman frente a discriminaciones que no pueden superarse mediante el aumento de la libertad contractual, y que requieren una respuesta desde otro campo, donde aparece el hombre como sujeto corporal, con derecho a alimentación, abrigo, vivienda, educación, salud, cultura, identidad, género, trabajo, etc. La segunda emancipación es una rebelión contra los efectos indirectos de los resultados de la primera emancipación, que librados a su lógica interna de compra-venta operan la inversión de los mismos derechos humanos y su sustitución por la institución mercado.

Esta dialéctica de la emancipación humana, que atraviesa la historia moderna, alcanza en nuestros días una situación crítica. En efecto, la estrategia de globalización en curso, motorizada a partir de los años ochenta, es una respuesta brutal e irracional desde arriba a la dinámica emancipatoria que procede desde abajo, desde la resistencia de los afectados contra los efectos destructivos de la lógica del mercado. Por aquellos años se había vuelto evidente la necesidad de fundar un nuevo 
orden económico y ecológico a nivel mundial, que limitara la racionalidad económica del mercado y le impusiera el respeto por la reproducción del hombre y de la naturaleza ${ }^{3}$. Sin embargo, la sociedad burguesa se negó entonces a afrontar ese desafío, prefirió negarlo y regresar a una forma de capitalismo puro - que había sido superado por la vía keynesiana después de la segunda guerra mundial-, cuya manifestación fue una política económica de tabula rasa. A partir de esta decisión, los ajustes estructurales realizados por los Estados, pero impulsados por las burocracias privadas, borraron los derechos humanos de la vida humana, dejando sin efecto conquistas históricas importantísimas, conseguidas a partir de las luchas seculares por la emancipación. Todos los derechos del ser humano como sujeto concreto fueron rápidamente anulados y marginados, sustituidos por la absolutización del mercado. No se trata, empero, de un regreso sin más a las declaraciones del siglo XVIII, pues el individuo propietario ha sido sustituido por las burocracias privadas, que, constituidas en un poder extraparlamentario de alcance mundial, dictan las políticas a los gobiernos, controlan los medios de comunicación y defienden su poder despótico en nombre de los derechos humanos. Su poder, no sometido al control público ni al voto democrático, ha socavado la democracia y ha convertido a los gobiernos en los apéndices del gran capital. "Se han transformado en la gran aplanadora del propio ser humano"4. En su entronización culmina la gran frustración de la modernidad: el hecho de que la emancipación desembocara en la dependencia más completa.

\section{El utopismo moderno y la necesidad de una crítica de la razón utópica}

¿Cómo es posible que la promesa moderna de emancipación haya conducido a su autosupresión? Para Hinkelammert, la explicación de esta paradoja se encuentra en la médula instrumental de la propia modernidad, en el tipo específico de racionalidad que promueve y privilegia la cultura moderna-occidental.

La dialéctica resistencia/institucionalización, que motoriza la lucha por la emancipación frente al poder de turno, es inherente a la condición humana y, como tal, está presente a lo largo de todo el recorrido del hombre en la tierra. Sin embargo, en el marco de la modernidad este proceso dialéctico corre serios riesgos de quebrarse. Ello se debe a que la sociedad moderna produce y privilegia un tipo específico de 
racionalidad, basada en el dominio científico y técnico del mundo y del hombre mismo, que le confiere al poder una capacidad de destrucción del entorno y de sojuzgamiento de las resistencias, de carácter inédito en la historia humana ${ }^{5}$.

La racionalidad moderna es una construcción histórico-cultural erigida a partir de una negación dialéctica de la cosmovisión antigua y medieval. Tal negación se manifiesta como secularización, como un proceso en el curso del cual los mundos trascendentes - los mitos de la reconciliación plena del hombre con Dios, con la naturaleza y con los otros hombres, en un ámbito "más allá" de esta vida- son reemplazados por mundos trascendentales, esto es, por idealizaciones construidas por abstracción y proyectadas al futuro como mecanismos de funcionamiento perfecto.

Importa destacar que esta sustitución de lo trascendente por lo trascendental, es decir, esta secularización de la racionalidad pre-moderna, se basa en una operación de abstracción, que dejará su impronta indeleble en la organización moderna de la experiencia del tiempo y de la sociedad. Abstracción significa que, frente a una realidad que no conforma, se erige un mundo "mejor posible", pensado y proyectado al futuro como un espacio "más allá" de este mundo. Su función es la orientación de la praxis hacia un horizonte de plenitud que, en sentido fáctico, no es realizable, pues es el resultado de una idealización, que ha sido construida a partir de la abstracción de los rasgos de finitud e imperfección que ostenta la condición humana real. El problema radica en que la razón moderna olvida el carácter trascendental de sus utopías, y, luego de proyectarlas al futuro, las considera como mecanismos de funcionamiento perfecto, fácticamente realizables a futuro.

En efecto, Hinkelammert considera que la Ilustración y las revoluciones burguesas sustituyeron el cielo religioso transmundano del cristianismo, no para abolirlo, sino para recuperarlo en una dimensión diferente: como conceptos abstractos de perfección, no concebidos como ideas reguladoras de la práctica —esto es, como una "totalidadausente", nunca presentificable en términos reales - sino como ámbitos de plenitud posible y alcanzable por un proceso de aproximación asintótica ${ }^{6}$. A estas proyecciones utópicas Hinkelammert las conceptualiza con la categoría de "totalidad presente". 
La escalera que une la tierra con este nuevo cielo secularizado es el mito del "progreso infinito, producto de una alianza entre tecnología y empresa, laboratorio y fábrica", que ha devenido en el fundamento de "una religión intramundana". El mito del progreso o del crecimiento indefinido introduce una trascendencia externa a la vida humana, a la que impone una tensión hacia el futuro, resultado de la proyección infinita de los desarrollos técnicos presentes.

Precisamente la construcción de utopías proyectadas al futuro y pensadas como modelos de perfección efectivamente alcanzables en el tiempo a partir de una aproximación asintótica, es una de las facetas de la dominación desplegada por el sujeto moderno sobre la naturaleza y sobre el otro social.

Desde esta perspectiva, la actual estrategia de acumulación capitalista que conocemos como "globalización" es el ulterior producto del ego imaginor moderno. Sus orígenes se remontan a los siglos XVI y XVII, cuando, a medida que las relaciones mercantiles se transformaron en relaciones capitalistas de producción y la lógica del cálculo de utilidades se impuso como racionalidad dominante, apareció y alcanzó hegemonía la ideología que plantea que el orden es resultado, no de un plan premeditado de los poderes públicos, sino de la autorregulación del mercado.

El mercado, especie de divinidad despojada de su carácter externo $y$ trascendente, fue convertido en un principio inmanente de funcionamiento perfecto, en una instancia reguladora omnisapiente que, a partir del choque de intereses particulares de actores individuales en conflicto, realiza automáticamente el interés general.

Sin embargo, muy pronto se hizo evidente que esta confianza mítica en la producción del orden a partir del desorden era completamente falaz. Se vio entonces que la orientación de la acción por el cálculo, cuando estaba librada a su propia lógica, producía efectos no intencionales de carácter destructivo, que, a la larga, socavaban las fuentes mismas de la riqueza: el ser humano y la naturaleza.

A medida que el desarrollo tecnológico acortó las distancias, "achicó" el planeta y aumentó su poder devastador, la destructividad de estos efectos involuntarios se generalizó, dando lugar a las crisis globales: particularmente, la pauperización creciente de la población, la alteración irreversible de la naturaleza y los problemas de violencia que afectan la 
convivencia humana. Estas crisis han alcanzado hoy tal magnitud, que ponen en riesgo las bases de sustentación de la vida misma a nivel del planeta, y nos muestran la evidencia palpable de que "el asesinato es un suicidio".

Como otras expresiones del pensamiento utópico moderno, la ideología neoliberal del mercado total es una utopía del progreso de aspiración universal, que promete la unidad del género humano alrededor de una institución sacralizada - el mercado- que funciona como mecanismo de legitimación del sistema social existente. Se trata de una utopía, que, en nombre de la Realpolitik, demoniza todas las demás utopías libertarias y se hace pasar por antiutopía. Proclama el "fin de la Historia”, desdeña abiertamente toda búsqueda de alternativas, y combate todas las formas de resistencia que se le oponen; de este modo, se socavan las posibilidades de frenar la irracionalidad que su lógica desencadena y de evitar el suicidio colectivo de la humanidad.

En este marco, la crítica de la razón utópica, que propone Hinkelammert, parte de considerar la proyección de utopías como una dimensión inevitable del pensamiento, que permite pensar lo imposible deseado y despejar, a partir de ello, el espacio de realización de lo posible. Por tanto no es, ella misma, antiutópica; lo que le importa iluminar es la falacia de la ilusión trascendental de que es presa la razón utópica cuando proyecta conceptos abstractos y los concibe como una realidad alcanzable. Cuando la razón utópica opera de este modo, la "totalidad" a la que se aspira es pensada como presente, en el sentido de alcanzable mediante una operación de acercamiento paulatino. Desde la mirada trascendental moderna todo lo que estorba la concreción de la perfecta idealización es percibido como factor distorsionante que debe ser suprimido, como "ruido" que empaña la transparencia de la plenitud posible. El resultado actual de ese procedimiento de aproximación asintótica a la utopía del mercado total es que "el conjunto de las condiciones de posibilidad de la vida humana aparece como una distorsión del mercado. Las mismas exigencias del circuito natural de la vida humana -el metabolismo entre el ser humano como ser natural y de la naturaleza circundante en la cual esta vida humana se desarrollason consideradas distorsiones del mercado [...]. Los propios derechos humanos son distorsiones del mercado desde el punto de vista de la lógica del mercado" 7 . 
La única forma de frenar este espiral de irracionalidad que nos lleva al suicidio colectivo es pensar la "totalidad" de otro modo, no como una presencia que está a la mano, a la vuelta del camino histórico, sino como "ausencia": indicio de la paradójica condición de un ser que aspira a la infinitud y a la transparencia, y tiene que realizarse en el marco de la finitud y la opacidad.

En las utopías respira un anhelo de totalidad, que es legítimo en tanto sólo la proyección de un ideal regulativo "imposible" permite dimensionar lo posible-real y juzgarlo a partir de la falta que impide una realización más plena. La dificultad reside en la conceptuación misma de la "totalidad", que determina el modo de aproximación a la utopía: por una parte, podemos concebirla como un horizonte siempre "ausente" que nos exige una aproximación práctica sujeta a permanente reformulación; por otra, podemos pensarla como una fórmula de perfección que está a la vuelta de la esquina y de la que sólo hay que apropiarse para hacerla "presente".

En este sentido, las utopías funcionan como detonadores de una dialéctica "totalidad-totalización". La totalidad proyectada surge siempre como respuesta e interpelación ante la totalización del sistema, que se reivindica a sí mismo como clausura de todo horizonte que pretenda trascenderlo. Ahora bien, en el primer caso, frente al sistema y a sus pretensiones de autosuficiencia, la totalidad se muestra como no lograda, como "ausencia-presente", como el síntoma de la falta que "grita" en los efectos no intencionales del sistema, como rebeldía ante la destrucción de la unidad hombre-naturaleza y hombre-hombre, que nos recuerda que la maximización de la lógica del cálculo destruye las bases de sustentación de la propia vida.

En el segundo caso, cuando la totalidad es concebida como disponible a la mano, el horizonte de posibilidad abierto por la orientación utópica resulta inmediatamente obturado. El resultado es la sacralización del statu quo, que, desde esa perspectiva, deviene o bien la meta ya alcanzada o bien una etapa en un camino que conduce, por aproximación asintótica, a una plenitud real. La utopía, en este caso, se vuelve funcional a la sociedad real, ya no la interpela, no devela la ausencia que gime en su seno; pierde por tanto, toda capacidad crítica.

Sólo si la utopía se piensa como idea de una "totalidad" que falta, se convierte en un instrumento que permite reclamar la plenitud hu- 
mana negada en la totalización real. En tal sentido, no se convierte a la ausencia de esa plenitud deseada en la meta futura de una aproximación asintótica, en un programa que se aspira a realizar o en una cosa que se quiere alcanzar - según el modelo medio-fin que constituye la modernidad como tal-, sino como "ausencia trascendental" que interpela y juzga ${ }^{8}$.

\section{Una ética de la resistencia y de la mediación}

La emancipación humana, entendida como un proceso dialéctico en el que la lucha por el reconocimiento de derechos cuaja en formas de institucionalidad, que cristalizan y oprimen al sujeto e impulsan de nuevo la resistencia, encuentra su punto de inflexión actual en el enfrentamiento del sujeto vivo con el mercado global, como sistema que lo aplasta. En este contexto, el bien común se expresa como exigencia de transformación del sistema. Sin embargo, esta exigencia no debe plantearse en términos de una abolición del sistema (mercado y dinero). En este punto, Hinkelammert desarrolla una visión crítica tanto de la utopía marxiana como de sus derivaciones en el pensamiento soviético posterior. Acuerda con Marx en la crítica a la sociedad capitalista en tanto "imposible" (en el sentido de insostenible): por su lógica destructora de las fuentes de la riqueza, es un tipo de sociedad incompatible con la reproducción de la vida humana. La afirmación de la vida como condición de posibilidad de cualquier sistema social viable, plantea la exigencia de producir un control consciente de la ley del valor. Sin embargo, suponer que las relaciones mercantiles pueden ser abolidas completamente es proyectar una ilusión utópica similar - inversa pero gobernada por la misma racionalidad instrumental moderna- a la del mercado total como mecanismo de funcionamiento perfecto?.

De la insostenibilidad del capitalismo se sigue la necesidad de la transformación social en un sentido socialista y democrático, acorde al fin de la sobrevivencia de la humanidad. Pero no se sigue la afirmación de un tipo particular de sociedad (ya sea la asociación de productores libres que coordinan directamente su división del trabajo, en Marx, o la coordinación a priori de todas las relaciones económicas, en el socialismo soviético). Y ello por dos razones complementarias, ambas derivadas de la concepción antropológica inherente al pensamiento de nuestro autor:

En primer lugar, la reproducción de la vida humana como criterio para juzgar cualquier estructura social y cualquier formulación ética, 
es un concepto negativo: exige no convalidar una determinada forma de organización social y política, en la medida en que ponga en riesgo la vida misma, pero no postula, en cambio, la necesidad de una forma de sociedad determinada El error que Hinkelammert le imputa a Marx consiste en haber convertido indebidamente ese criterio negativo en positivo, tal como sucede cuando se proyecta una societas perfecta como única alternativa posible. En este mecanismo opera la razón utópica moderna, no sometida a crítica, que plantea de nuevo una meta a alcanzar en términos de aproximación asintótica a un fin de la historia predeterminado e hipostasiado, que juzga sobre el presente en términos maniqueos de condena o sacralización absolutas.

En segundo lugar, las relaciones mercantiles y el cálculo en dinero, que "están sofocando la vida humana", no pueden, empero, ser abolidos porque su surgimiento no se debe exclusivamente a la aparición contingente de la propiedad privada. Además de esta razón histórica existe una explicación de la aparición del mercado y del dinero que remite a la condición finita y fragmentaria — no superable - del conocimiento humano mismo ${ }^{10}$.

En efecto, la coordinación de la división social del trabajo, en tanto es un producto de la práctica social e histórica de los hombres, está sujeta a las propias limitaciones de nuestra capacidad cognitiva. A partir de una cierto grado de complejidad social, el desarrollo de la división social del trabajo, como sistema interdependiente en todos sus componentes, deja de ser susceptible de una coordinación directa y a priori, basada en el conocimiento suficiente de todas las variables puestas en juego. Las relaciones mercantiles suplen esta limitación del conocimiento, produciendo una coordinación a posteriori e indirecta del sistema. Cuanto más avanza la división social del trabajo es menos reversible la institucionalización de las interrelaciones por el mercado, que supondría un regreso a sistemas de producción anteriores y menos eficientes en términos de productibilidad del trabajo.

Para Hinkelammert, la exigencia de superar el capitalismo surge a partir de la evidencia de los desastres que produce el automatismo del mercado. De allí se plantea la necesidad de una adecuada planificación económica, que asegure la racionalidad a partir de una división social del trabajo, capaz de solventar las necesidades. Esta es la finalidad de la planificación económica, que garantiza la autonomía de decisión del sujeto humano respecto de la distribución de los ingresos y del empleo; pero 
esta necesidad no debe plantearse en términos de una polarización excluyente y maniquea entre mercado y plan.

La reproducción de la vida de todos, en tanto condición de posibilidad de cualquier horizonte de emancipación humana, es el criterio que debe decidir la sobre la delimitación entre mercado y plan, en cada situación histórica particular. Sin embargo, ello no significa que la satisfacción de las necesidades básicas constituya, en sí misma, la meta de la emancipación. Es, más bien, la condición material previa para que la emancipación sea posible y, en tal sentido, constituye su piso, no su techo. En cualquier sociedad histórica se plantea una tensión entre el nivel de satisfacción de las necesidades institucionalmente organizado, y la proyección de una satisfacción posible, más plena y abarcativa, que impulsa la crítica de las instituciones existentes. Por eso, la satisfacción de las necesidades básicas no es estática, sino que se desarrolla en el proceso histórico mismo. Y la dinámica de ese proceso procede de la imaginación trascendental, por la cual la idealización de la satisfacción subjetiva de las necesidades plantea la necesidad de reformular y cambiar las instituciones dadas. Esa puesta en movimiento de la historia nace siempre del reconocimiento entre sujetos como tales, un reconocimiento que excede el marco institucional, rompe sus límites y orienta la praxis hacia la universalidad humana trascendental, en el sentido de la eliminación de las opresiones, discriminaciones y explotaciones. Sin embargo, las emancipaciones que brotan de este suelo requieren una respuesta institucional para afirmarse y generalizarse. Sin instituciones y leyes no es posible la satisfacción de las necesidades humanas.

La dominación se expresa en leyes e instituciones, y conforma una determinada legalidad. Pero legalidad no es sinónimo de legitimidad. La ley sólo es legítima en la medida en que está al servicio del despliegue de las potencialidades humanas, para lo cual debe, como mínimo, resultar compatible con la sobrevivencia de la humanidad y de la naturaleza. Por su propia inercia, todo sistema de leyes e instituciones tiende a la irracionalidad, a su sacralización y a la subordinación del hombre y el medio natural a sus propios fines. En el otro extremo, la exigencia de legitimidad y de racionalidad procede siempre de la resistencia frente al sistema. 


\section{La emancipación humana hoy: el espacio de la ciudadanía civil}

La ética del bien común supone adoptar la perspectiva de la institucionalización de la satisfacción de las necesidades humanas, que despeja la consideración de un tipo de sujetividad humana diferente al individuo privado y al ciudadano político. Frente a ambas abstracciones, se trata de atender al sujeto vivo, dotado de cuerpo y de determinaciones concretas, que, para no morir, requiere una institucionalidad que asegure la posibilidad de su desarrollo, la efectiva realización de un proyecto de vida en el marco de condiciones materiales garantizadas por instituciones.

En este sentido, Hinkelammert propone un tipo de democracia que se atreve de calificar como "socialista", y a la que concibe como deudora de las dos emancipaciones humanas desplegadas a partir de las revoluciones burguesas. No abreva solamente en el pensamiento de Marx, al que, empero, reconoce como fuente ineludible, sino también en una línea del pensamiento liberal-racionalista, representada especialmente por Rousseau. Este liberalismo, que nuestro autor llama "de la soberanía popular", nace en pugna con el liberalismo antisubjetivo de Locke, cuya expresión actual es el neoliberalismo.

Hinkelammert considera que, después de la experiencia histórica del socialismo real, es necesario actualizar el liberalismo racionalista de la soberanía popular — para él, operante en el propio Marx ${ }^{11}$ - de una manera nueva, articulándolo a la crítica del socialismo que no logró limitar la actividad estatal en función del sujeto.

Su propuesta consiste, por tanto, en recuperar, junto con la crítica a la lógica mercantil de cuño marxiano, la categoría rousseauniana de ciudadanía, sometiendo el carácter ficcional de esta última a una crítica determinada. Pues, si el "ciudadano" despeja un horizonte de libertad, se debe a la peculiar relación que entabla con la ley. En cuanto produce la ley con su libre consentimiento, no es subsumido por ella y permanece como instancia que la legitima y que puede interpelarla en la medida en que esta tienda a independizarse de su origen sujetivo. El problema aparece cuando Rousseau ahoga este primer impulso libertario del concepto de ciudadanía en el concepto de "voluntad general". La voluntad general opera en sentido inverso a la afirmación la libertad del sujeto frente a la ley: es un principio absoluto que, al someter al ciudadano, funge como anterior a cualquier decisión o elección efecti- 
va. "El resultado es una legalidad que predetermina lo que el ciudadano tiene que afirmar libremente, si quiere ser libre [...]. La voluntad general es considerada una voluntad a priori a la libertad del ciudadano, y la voluntad de todos tiene que sometérsele"12. Por este camino, el descubrimiento de Rousseau se diluye, y la libertad del ciudadano, subsumida en un imperativo anterior a la voluntad del sujeto concreto, termina equivaliendo a la ley de la igualdad contractual: todos son iguales y libres en la medida en que se someten a la lógica contractual, de donde resulta que la libertad no es más que subordinación del hombre al mercado.

Para conjugar la crítica de Marx a la dominación-explotación de la sociedad capitalista y la idea de la libertad de Rousseau como autodeterminación, hay que, en primer lugar, retrotraer el concepto de ciudadanía antes de su deriva por la voluntad general. La recuperación de un espacio para una libertad e igualdad no abstractas, sino preñadas de determinaciones concretas, supone sustituir el concepto de voluntad general, que hace abstracción de las diferencias como determinaciones concretas y plantea una ciudadanía política despojada de corporalidad, y oponerle una voluntad de todos, entendida como espacio de la ciudadanía civil, que reúne en sí misma el carácter social, natural e individual del sujeto, y permite pensar la legitimidad de nuevos derechos, que dan pie a las luchas de la segunda emancipación.

La articulación de un espacio para el ejercicio de la ciudadanía civil, desde donde los sujetos concretos, no despojados de sus atributos, resistan los efectos destructivos de la primera emancipación, interpelen a las instituciones y exijan el control consciente de la ley del valor y el control democrático de la dominación política, debe alcanzar una capacidad suficiente para intervenir en la lógica inerte del sistema y someterla a reglas de funcionamiento establecidas desde la afirmación de la vida.

\section{Conclusión}

Por esta vía, la reflexión de Hinkelammert se devela como una respuesta inquietante dentro del campo de la filosofía política actual; una respuesta que se atreve a rescatar las potencialidades libertarias de la categoría de ciudadanía, sin desconocer sus límites teóricos e ideológicos, pero la reenvía hacia una formulación de la sujetividad de carácter fundante, no constreñida dentro de las fronteras teóricas de la socie- 
dad burguesa en general y de su configuración histórica actual (el capitalismo global).

Por una parte, el filósofo logra escapar así a las trampas del reformismo y a sus ilusiones de una democracia acotada y vaciada de contenido; nos muestra que éste permanece preso de la interpretación liberal-abstracta del sujeto político, sumiso a la lógica de la razón calculante y de la primacía del mercado, y nos plantea la exigencia de recuperar el horizonte de la afirmación de la libertad y dignidad humanas en toda su necesaria amplitud y actualidad.

Por otra parte, nos plantea la necesidad de someter a una profunda autocrítica las utopías emancipatorias que elaboraron las generaciones pasadas y de redefinir un proyecto de liberación que no evada el reconocimiento de los límites de opacidad y finitud que son intrínsecos a la condición humana.

En el marco de la fragmentación de las sociedades capitalistas actuales y de la cultura de la desesperanza característica de nuestra época, se hace necesario resaltar la importancia de la dimensión utópica. Hoy más que nunca es necesario pensar las posibilidades de la realidad a partir de un ideal imposible que abra una brecha hacia las transformaciones posibles. Pero es igualmente necesario reconocer el carácter trascendental y no empírico de ese ideal regulativo.

La intervención de la crítica, en este contexto, significa desacralizar el sistema e historizarlo, mostrar su génesis y su desarrollo histórico para poder pensarlo como producto de la praxis humana y, por tanto, no ajeno a su posible transformación. Significa también volcar una luz sobre los efectos de la lógica capitalista, que destruye las fuentes de la riqueza, subordina la humanidad del productor a los imperativos instrumentales de la reproducción del sistema y reduce la naturaleza a producto de consumo y objeto de explotación. La crítica devela, en tal sentido, la herida mortal producida sobre el cuerpo del "circuito natural de la vida humana", como consecuencia de la cual se ha establecido una contradicción agónica entre el producto de la actividad práctica (el trabajo objetivado o muerto) y las fuentes de la riqueza (el trabajo vivo y el metabolismo hombre-naturaleza como condición de posibilidad de la vida misma).

En definitiva, someter a crítica la totalización del sistema y su proyección sacralizadora como "totalidad-presente", significa alumbrar la condición humana como infinitud que se desarrolla en el marco de la 
finitud y que encierra, por tanto, la necesidad de reconocer el carácter trascendental de las utopías.

\section{Abreviaturas de las obras de Hinkelammert citadas en este trabajo:}

RSR: El retorno del sujeto reprimido, Bogotá, Universidad Nacional de Colombia, 2002.

CEySSE: Cultura de la esperanza y sociedad sin exclusión, San José, DEI, 1995.

$C R U$ : Crítica de la razón utópica, Bilbao, Desclée de Brouwer y Junta de Andalucía, 2002.

DCS: Determinismo, caos, sujeto. El mapa del emperador, San José, DEI, 1996.

\section{Notas}

1. Cfr. F. Hinkelammert, "La negativa a los valores de la emancipación humana y la recuperación del bien común", $R S R, 79-89$.

2. Cfr. F. Hinkelammert, "La inversión de los derechos humanos: el caso de John Locke”, RSR, 61 ss.

3. Cfr. F. Hinkelammert, "El mercado como sistema autorregulado y la crítica de Marx", $R S R, 169-171$

4. F. Hinkelammert, "La rebelión en la tierra y la rebelión en el cielo: el ser humano como sujeto", $R S R, 319$. Sobre las "burocracias privadas, Cfr. "La crisis de poder de las burocracias privadas. El socavamiento de los derechos humanos en

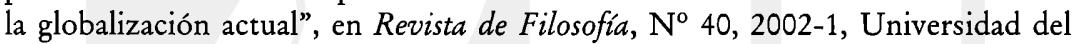
Zulia, Maracaibo, 15-33.

5. Hinkelammert sostiene: "por primera vez tenemos la perspectiva de una catástrofe apocalíptica, la cual no será provocada por un jinete montado sobre un caballo blanco, sino producida por nosotros mismos. Nunca antes hubo algo semejante en la historia. Estamos frente al problema del suicidio colectivo de la humanidad. Hubo suicidios colectivos, así como mitos del suicidio colectivo de la humanidad. Sin embargo, después de la Segunda Guerra Mundial, la posibilidad fáctica de un suicidio colectivo de la humanidad la tenemos hoy por primera vez": "Utopismos y utopías de la modernidad. Acerca de la Crítica a la razón utópica. Entrevista de Norbert Arntz", en Itinerarios de la razón crítica. Homenaje a Franz Hinkelammert en sus 70 años, San José, DEI, 2001, 77.

6. "Los que exigen que la realidad se aproxime a estas idealizaciones, nunca sostienen que se las pueda alcanzar efectivamente. Pero tienen que pronunciarse sobre la relación entre la realidad y su ideal, expresado por un concepto de mecanismo de funcionamiento perfecto. En la ciencia moderna, lo usual es describir esta relación como una aproximación asintótica infinita, un concepto tomado de la matemática. [...] Se habla de curvas asintóticas, cuando una curva se aproxima cada vez más a un valor fijo, sin alcanzarlo nunca completamente. Cada punto de la curva está distante del valor fijo, al cual se aproxima, aunque la distancia se

Crítica y utopia en la fllosofía de Franz Hinkelammert 
haga infinitamente pequeña. [Puede] decirse que la curva llega a alcanzar el valor fijo, al cual se aproxima, en el infinito"; en F. Hinkelammert, "Sobre la concepción de sociedades perfectas en la metodología de las ciencias económicas", inéd., San José, 2000, 22 p., 5.

7. Franz Hinkelammert, CEySSE, $278 \mathrm{~s}$.

8. El término "trascendental" remite al pensamiento de Kant y, de modo particular, a su consideración de la razón como origen de principios incondicionados (no empíricos), que no surgen de la experiencia ni encuentran en ella una referencia inmediata, pero que remiten a la totalidad de las condiciones de toda experiencia posible. La razón, a través de sus ideas, puede conceptuar lo incondicionado, como aquel conjunto de condiciones que comprende toda experiencia pero que no puede ser objeto de experiencia. Cfr. I. Kant, Crítica de la razón pura, México, Porrúa, 1977, 168-174; y F. Hinkelammert, CRU.

9. Para Hinkelammert, "el problema en la teoría de Marx no descansa precisamente sobre su análisis del capitalismo [...] sino en su imaginación de una alternativa [...]. Cuando Marx describe al capitalismo como un orden que surge como reacción al desorden producido por este mismo capitalismo, da sin duda una imagen realista y objetiva del capitalismo. Pese a eso, Marx sigue preso de la ilusión trascendental de un orden apriorístico y determinista. Por eso la alternativa al capitalismo que él esboza — socialismo o comunismo, asociación de productores libres o Robinson social— designa también un orden determinista [..., que] debe sustituir a un orden que surge como reacción a desórdenes [...]. De esta visión esquemática surge el socialismo soviético, que quiere realizar por medio de un plan central un tal determinismo del orden. Si bien Marx no concibe algo parecido a una planificación central, elabora la ilusión trascendental correspondiente". Esto sucede, según el autor, cuando Marx insinúa la superioridad de una coordinación a priori de la división social del trabajo, producida conscientemente por los productores, en relación con el equilibrio compulsivo, impuesto a posteriori por el mercado a espaldas de los productores, mediante el mutismo de los cambios de los precios de las mercancías (Cfr. C. Marx, El Capital, I, ed. cit., 289s). Prosigue Hinkelammert: "Tomada la imagen de una coordinación a priori como una meta por hacer, por realizar efectivamente, y no apenas como una referencia o una especie de 'idea regulativa', se transforma en una ilusión trascendental [...]. Sin embargo, toda constitución de un orden apriorístico y determinista es imposible y jamás resultará, porque aspira más allá de lo que es la conditio bumana [...]. No se trata de [realizar] una libertad a priori de leyes que se imponen a espaldas de los actores, como pudo ser entendido el análisis de Marx, sino de un conflicto continuo y constante para disolver las fuerzas compulsivas de los hechos —en el grado que sea posible- por medio de la acción asociativa o solidaria"; en F. Hinkelammert, "Determinismo y autoconstitución del sujeto: las leyes que se imponen a espaldas de los actores y el orden por el desorden", DCS, 252-259.

10. Cfr. F. Hinkelammert, "Leyes universales, institucionalidad y libertad: el sujeto humano y la reproducción de la vida real, CRU, 327-332.

11. Hinkelammert encuentra una expresión paradigmática de la inscripción libertaria del pensamiento de Marx, en esta cita de El capital (ed. cit., I, 606): "Y frente a la vieja reina de los mares se alza, amenazadora y cada día más temible, la joven 
república gigantesca: 'Un duro destino atormenta a los romanos, la maldición por el asesinato del hermano? (Horacio)". Roma, símbolo del imperio en todos los tiempos, es la Inglaterra de la época de Marx (la "reina de los mares") y los Estados Unidos de la nuestra. Marx denuncia el asesinato del hermano como fundante de toda civilización imperial y anuncia el levantamiento, frente al poder absoluto del imperio de turno, de "la joven república gigantesca": "es la sociedad civil desde abajo - interpreta Hinkelammert-, cuando logra hacerse democracia vigente". Este Marx libertario permanece a la orden del día y señala "la tarea de hoy, en la cual estamos empeñados con el movimiento que se enfrenta a la actual estrategia de globalización: recuperar la libertad de opinión, recuperar la capacidad del ciudadano para controlar a las burocracias privadas de las empresas transnacionales y poner la economía al servicio de la vida humana y de toda la naturaleza"; F. Hinkelammert, "El nuevo imperio y nosotros", en Pasos. Segunda época, San José, DEI, $\mathrm{N}^{\circ}$ 107, mayo-junio de 2003, 47. 12 F. Hinkelammert, "La negativa a los valores de la emancipación humana...", $R S R, 100-101$. 